\title{
Ray Woosley
}

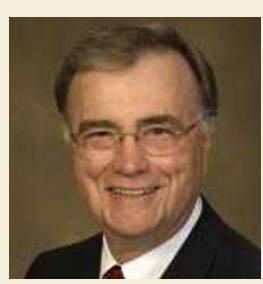

President and CEO, The Critical Path Institute (C-Path), Tucson, Arizona and Rockville, Maryland, USA. In January 2005, Ray Woosley, M.D., Ph.D., became President of C-Path formed by the FDA, SRI International and the University of Arizona to accelerate the development of safe innovative medicines. Before then, Woosley was Vice President for Health Sciences at the University of Arizona and Dean of the College of Medicine (2001-2005).

What led you to found C-Path and what have been the successes so far?

While Mark McClellan was Commissioner at the FDA, then Deputy Commissioner Janet Woodcock launched the Critical Path Initiative, which included a call for collaborators and partnerships. It was clear to me that to make progress we needed to bring the FDA and the industry together in a constructive relationship that would allow the sharing of information to improve the process for developing safe innovative drugs. I convinced the University of Arizona that to achieve this we should form an independent, non-profit organization with the sole mission to partner with the FDA. C-Path was only possible because of Woodcock's commitment to institute science-based change, supported by McClellan and the Commissioners that followed. Through the Arizona community of governments and foundations we were able to obtain the funding to create this neutral entity.

In its first 3 years, C-Path has made important small steps to establish a process for changing drug development. Looking at these steps in aggregate, the biggest change has been our demonstration that companies and the regulatory bodies can work together. To enable data sharing, a major accomplishment was the recognition of the need for the execution of a legal document that covers intellectual property, confidentiality, liability and anti-trust issues. We also worked with the FDA and the EMEA to create a new pathway for submission of new scientific information as part of an application for regulatory changes - we brought together 17 companies to present scientific data on improved safety testing methods to the scientists from both agencies. C-Path's Predictive Safety Testing Consortium (PSTC) recently used this process, and the FDA and EMEA jointly declared seven new biomarkers of drug-induced kidney injury 'qualified for use' in drug development.
Could you describe C-Path's role in last year's recommendation that doctors should consider a genetic test when first prescribing warfarin? The FDA was in a difficult situation because they were aware of research that indicated that genetic tests might improve the outcomes with warfarin, a complex drug that has many risks to patients if the dose is too high or low. But as warfarin is a generic drug there was no corporate entity willing to do the confirmatory science and request a revised drug label. To help address this, we asked academic scientists to help provide the expertise and data needed to enable the FDA to revise the label and include information on the value of genetic tests when selecting initial doses of warfarin. There is still a lot left to be done, but our role was - and continues to be - as an independent convener, identifying consensus and pulling together the additional data required.

\section{Is C-Path working with companies and the FDA on any other tests at the present time?} We are working closely with the FDA and the National Cancer Institute (NCI) to find a better path for biomarkers to be qualified for use as diagnostic tests for both regulatory and clinical decision making. During drug development of targeted therapies, sponsors need to be able to co-develop a drug and diagnostic test to predict how a patient will respond to the drug, as well as to facilitate determination of the drug's efficacy. Our Chief Scientist Jeffrey Cossman and his team have brought together diagnostics companies to share their methodologies and design an $\mathrm{NCI}$ trial to determine whether the epidermal growth factor receptor is a biomarker that can predict a patient's response to targeted therapy for lung cancer. We are also working with the FDA and diagnostics companies to create a new entity that would independently certify the performance characteristics of diagnostic tests, as no such body currently exists.
How will C-Path help the FDA with its recent mandate to increase drug-safety monitoring through the Sentinel Initiative?

C-Path serves as one of the 14 federally funded Centers for Education and Research on Therapeutics (CERTs) working with the FDA to improve safety surveillance systems. Each of the CERTs has focused on a different aspect of drug or medical product safety and I think we all conclude that there is no simple solution to the problem; multiple tools and approaches will be required. We are currently evaluating tools available in other countries, for example the UK yellow-card system. Although the electronic medical record holds great promise, one of the real problems is that there are limitations and discrepancies of electronic databases such as those that signal potential drug interactions. Improving the quality of this data will be a significant challenge.

\section{Given that C-Path is an independent non-profit institute, what are the challenges for obtaining the money you need?} So far, we have been able to address this without asking the usual stakeholders for support. If we accept funds from our commercial stakeholders, such as pharmaceutical, diagnostic or health-insurance companies, then we would risk the perceived loss of neutrality and our ability to be a trusted third party. The ultimate stakeholder therefore is the public and we have been very fortunate to find such support in Arizona. The governor and state legislators recognize the importance of developing a 'knowledge economy', including investment in education and biotechnology. The state and leading foundations also see that for technology to reach the public, and for it to have economic value to the state, it has to be approved by the FDA, and C-Path is recognized as an important component of this.

Although independent funding is important to seed our projects, it is not the only resource available. For example, in the PSTC the industry members and FDA spend much more in-kind than we can invest. However, our core neutral funding ensures that the FDA and EMEA can join us and industry in these scientific discussions and have confidence that our only purpose is to serve the public health. 